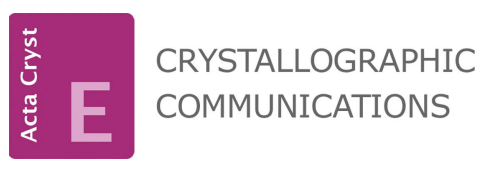

ISSN 2056-9890

Received 4 December 2017

Accepted 17 January 2018

Edited by P. Bombicz, Hungarian Academy of Sciences, Hungary

Keywords: crystal structure; medium-sized heterocycles; bisphosphonothioyl disulfanes; $\mathrm{C}-\mathrm{H} \cdots \mathrm{S}=\mathrm{P}$ interactions.

CCDC references: 1558043; 719124

Supporting information: this article has supporting information at journals.iucr.org/e

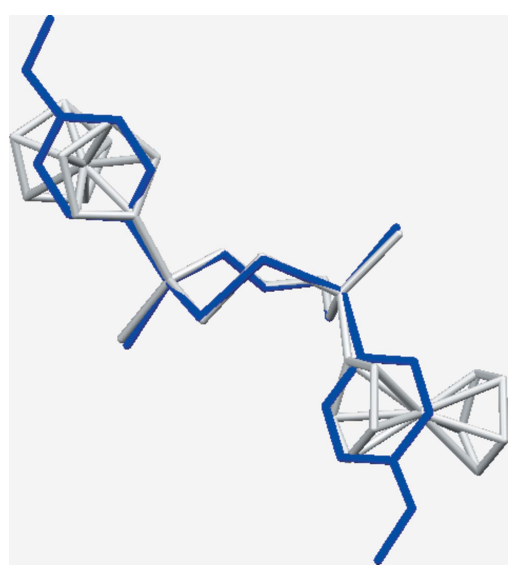

OPEN $\odot$ ACCESS

\section{Crystal structures of eight- and ten-membered cyclic bisanisylphosphonothioyl disulfanes and comparison with their $\boldsymbol{P}$-ferrocenyl analogues}

\author{
Witold Przychodzeń ${ }^{\mathrm{a}}$ and Jarosław Chojnacki ${ }^{\mathrm{b} *}$ \\ aDepartment of Organic Chemistry, Gdańsk University of Technology, G.Narutowicza 11/12, 80233-PL, Gdańsk, \\ Poland, and ${ }^{\mathbf{b}}$ Department of Inorganic Chemistry, Gdańsk University of Technology, G.Narutowicza 11/12, 80233-PL, \\ Gdańsk, Poland. *Correspondence e-mail: jaroslaw.chojnacki@pg.edu.pl
}

Two new crystal structures of eight- and ten-membered cyclic bisanisylphosphonothioyl disulfanes, namely 2,5-bis(4-methoxyphenyl)-1,6,3,4,2 $\lambda^{5}, 5 \lambda^{5}$ dioxadithiadiphosphocane-2,5-dithione, $\mathrm{C}_{16} \mathrm{H}_{18} \mathrm{O}_{4} \mathrm{P}_{2} \mathrm{~S}_{4}$, and 2,5-bis(4-methoxyphenyl)-1,6,3,4,2 $\lambda^{5}, 5 \lambda^{5}$-dioxadithiadiphosphecane-2,5-dithione, $\mathrm{C}_{18} \mathrm{H}_{22} \mathrm{O}_{4} \mathrm{P}_{2} \mathrm{~S}_{4}$, have been determined and compared to structures of the ferrocenyl analogues. The eight-membered rings have similar conformations (TBC) but the tenmembered macrocycles are differently puckered. Structural parameters of the relevant SPSSPS motif have been analysed and are discussed in detail. Compound $\mathbf{1}$ was refined as an inversion twin and $\mathbf{2}$ was refined as a twocomponent rotational twin.

\section{Chemical context}

The most widely used sulfur-transfer agents for thionation of carbonyl compounds are the four-membered 2,4-dianisyl-1,3dithiadiphosphetane disulfide dimer $[\operatorname{AnP}(\mu-\mathrm{S}) \mathrm{S}]_{2}$ and the 2,4-diferrocenyl-1,3-dithiadiphosphetane disulfide dimer $[\mathrm{FcP}(\mu-\mathrm{S}) \mathrm{S}]_{2}$, i.e. Lawesson reagent LR (Jesberger et al., 2003) and ferrocenyl Lawesson reagent fLR (Foreman et al., 1996). However, thiophosphine oxides (AnPSO or FcPSO) separating as cyclic trimers during thionation reactions are usually unwanted side-products. On the other hand, the corresponding alkoxyphosphinodithioic acids, i.e. $\mathrm{An}(R \mathrm{O}) \mathrm{P}(\mathrm{S}) \mathrm{SH}$ and $\mathrm{Fc}(R \mathrm{O}) \mathrm{P}(\mathrm{S}) \mathrm{SH}$, obtained in a simple reaction between LR or fLR and alcohols, are of considerable interest because they form a plethora of structurally interesting chelate complexes with metal ions (van Zyl \& Woollins, 2013).

The reactions between Lawesson's reagent and diols/ diphenols have been successfully involved in the preparation of bis(anisylphosphonodithioic) acid derivatives and among them the unique eight-, nine- and ten-membered cyclic bisanisylphosphonothioyl disulfanes (Przychodzeń, 2004). A high-yielding formation of these medium-sized cyclic disulfanes upon oxidation of bis(anisylphosphonodithtioic) acid salts by iodine proceeding without oligomeric by-products may be attributed to their fixed structure, containing the most preferred a zigzag motif of the SPSSPS unit. Slightly modified procedures with respect to the original method have recently been applied for the synthesis of related cyclic bis(ferrocenylphosphonothioyl)disulfanes, e.g. eight-membered $\mathbf{1 a}$ (Pillay et al., 2015) and ten-membered $2 \boldsymbol{a}$ (Hua et al., 2017) and their crystal structures have been determined. Here we report 
crystal structures compounds $\mathbf{1}$ and $\mathbf{2}$, containing anisyl groups instead of the ferrocenyl moiety.
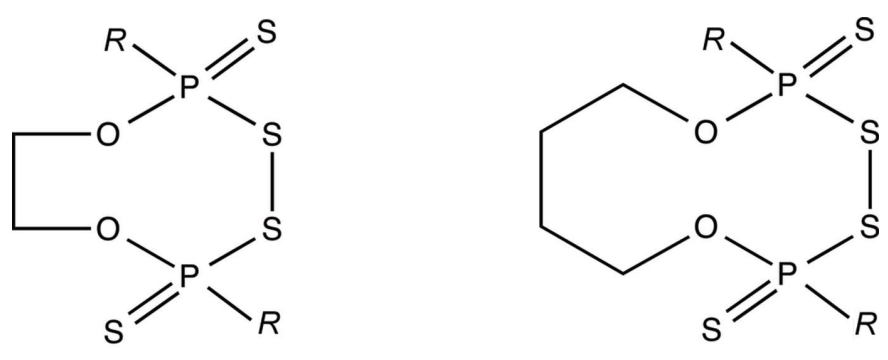

$$
\begin{aligned}
& 1 R=A n \\
& 1 a R=F c
\end{aligned}
$$$$
2 R=\text { An }
$$$$
\text { 2a } R=\text { Fc }
$$
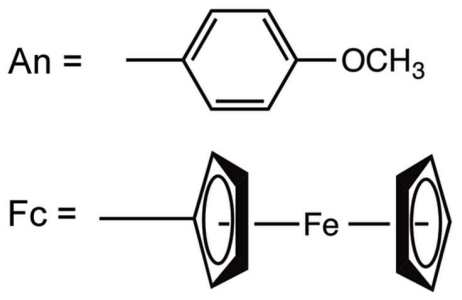

\section{Structural commentary}

Views of molecular structures and atom-labeling scheme for $\mathbf{1}$ and $\mathbf{2}$ are given in Figs. 1 and 2, respectively. Compound $\mathbf{1}$ crystallizes in the $P_{3} 2_{1} 2$ space group with a half-molecule in the asymmetric unit. It follows that the molecule obeys point group symmetry described by Schoenflies symbol $C_{2}$ (or symbol 2 in international notation). The related ferrocenyl compound $\mathbf{1} \boldsymbol{a}$ crystallizes in space group $C 2 / c$ with non-typical three and half independent molecules in the asymmetric unit $(Z=28)$, which complicates comparisons.

Compound 2 forms a monoclinic crystalline phase obeying $P 2_{1} / c$ space-group symmetry with one molecule in the asymmetric unit and $Z=4$. The related ferrocenyl structure $\mathbf{2 a}$ crystallizes in space group $P \overline{1}$ with one molecule in the asymmetric unit.

The anisyl groups as well as the ferrocenyl groups on the two phosphorus atoms are positioned in a trans arrangement,

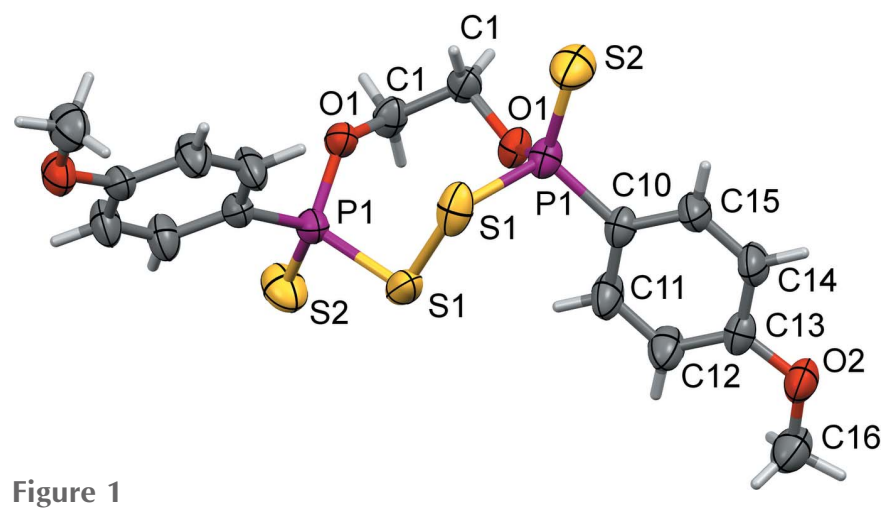

The molecular structure of $\mathbf{1}$, showing the atom-labelling scheme. Displacement ellipsoids are drawn at the $50 \%$ probability level. Symmetry-equivalent atoms are generated by the operation $(y+1$, $x-1,-z)$.

i.e. above and below the macrocycle ring plane for all compounds 1-2a, which is also typical for all open-chain bisphosphorothioyl disulfanes studied previously (Gray et al., 2004).

The S-S bond lengths have values of 2.068 (2) $\AA$ for $\mathbf{1}$; 2.0697 (10), 2.0704 (10), 2.0685 (10), 2.0711 (15) $\AA$ for $\mathbf{1 a}$; 2.074 (3) $\AA$ for 2 and 2.0788 (9) $\AA$ for $2 \boldsymbol{a}$. They are longer than the typical $\mathrm{S}-\mathrm{S}$ bond lengths for known diorganyl disulfanes $R \operatorname{SS} R[2.05$ (3) $\AA$ ] . The observed $\mathrm{S}-\mathrm{S}$ bond elongation in 1$\mathbf{2 a}$ may be correlated with the PSSP torsion angles (Knopik et al., 1993). As expected, exocyclic $\mathrm{P}=\mathrm{S}$ bond lengths ( $c a$ $1.92 \AA)$ are shorter than the endocyclic $\mathrm{P}-\mathrm{S}$ bonds ( $c a$ $2.10 \AA)$.

All phosphorus atoms in 1-2a adopt a distorted tetrahedral geometry, where the $\mathrm{C}-\mathrm{P}=\mathrm{S}$ angles deviated the most $\left(116.1-118.5^{\circ}\right)$ from the ideal tetrahedral angle. This is obviously due to the steric effects of the anisyl and ferrocenyl substituents. On the other hand, it is worthy to note that the $\mathrm{O}-\mathrm{P}-\mathrm{S}$ bond angles in 1-2a $\left(107-108^{\circ}\right)$ are not distorted, probably due to minimal conformational strain present in those medium-sized heterocycles. Moreover, both the $\mathrm{P}=\mathrm{S}$ and aromatic anisyl groups in $\mathbf{1}$ are almost perfectly coplanar (unlike $\mathrm{P}=\mathrm{S}$ and the cyclopentadienyl ring in $\mathbf{1 a}$ ), which provides energetically favorable conjugation [torsion angle $\mathrm{S} 2-\mathrm{P} 1-\mathrm{C} 10-\mathrm{C} 15=-3.8(4)^{\circ}$ in $\mathbf{1}$ vs $35.75(3)^{\circ}$ for the equivalent angle in a selected representative molecule with $\mathrm{Fe} 7$ in $\mathbf{1 a}$ ]. The other related independent torsion angles in $\mathbf{1 a}$ are -31. (3), $-33.9(3), \quad-27.0(3), \quad-28.7(3), 34.8(3)$, $35.7(3)^{\circ}$, for $\mathrm{Fe} 1-\mathrm{Fe} 6$, respectively.

It is well recognised that PSSP torsion is a characteristic feature of all disulfanes as a class of organic compounds. The structure of $\mathbf{1}$ is the most symmetric with the lowest PSSP torsion $\left[-93.68(8)^{\circ}\right]$ and shows only a moderate deviation from a right angle. The PSSP torsion angles in $\mathbf{1} a[-101.19$ (4), $-100.06(4),-101.47(4)$ and $\left.99.89(4)^{\circ}\right]$ are $6-8^{\circ}$ wider than in 1. Notably, ten-membered disulfanes have even wider PSSP torsion angles and the difference between them is smaller, -112.89 (11) and $114.9(4)^{\circ}$, for $\mathbf{2}$ and $\mathbf{2 a}$, respectively.

Only non-classical hydrogen-bonding interactions of the type $\mathrm{C}-\mathrm{H} \cdots X(X=\mathrm{O}$ or $\mathrm{S})$ can be found in the structures of 1 and $\mathbf{2}$ (Tables 1 and 2).

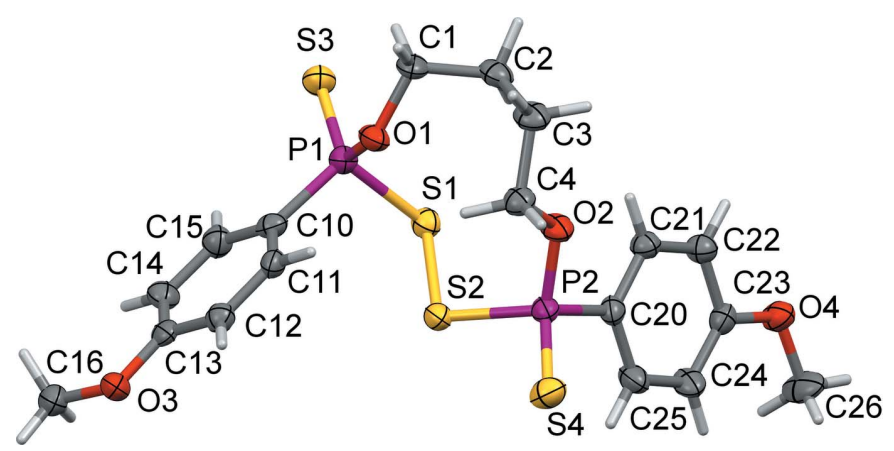

Figure 2

View of the asymmetric unit of $\mathbf{2}$, showing the atom-labelling scheme. Displacement ellipsoids are drawn at the $50 \%$ probability level. 
Table 1

Hydrogen-bond geometry $\left(\AA{ }^{\circ}\right)$ for $\mathbf{1}$.

\begin{tabular}{lllll}
\hline$D-\mathrm{H} \cdots A$ & $D-\mathrm{H}$ & $\mathrm{H} \cdots A$ & $D \cdots A$ & $D-\mathrm{H} \cdots A$ \\
\hline $\mathrm{C} 1-\mathrm{H} 1 A \cdots \mathrm{O} 2^{\mathrm{i}}$ & 0.97 & 2.60 & $3.4843(2)$ & 151 \\
$\mathrm{C} 14-\mathrm{H} 14 \cdots \mathrm{O}{ }^{\mathrm{ii}}$ & 0.93 & 2.55 & $3.4548(2)$ & 163 \\
\hline
\end{tabular}

Symmetry codes: (i) $x+\frac{1}{2},-y+\frac{1}{2},-z+\frac{1}{4}$; (ii) $x-\frac{1}{2},-y+\frac{1}{2},-z+\frac{1}{4}$.

The transannular P...P distances are very similar within the same ring size and increase, from 4.3331 (17) $\AA$ in $\mathbf{1}$ and 4.2625 (9), 4.2670 (9), 4.2652 (9) or 4.261 (1) $\AA$ (for different independent molecules in $\mathbf{1 a}$ ) for eight-membered rings, to 4.614 (2) in $\mathbf{2}$ and 4.604 (1) $\AA$ in $\mathbf{2 a}$ for the ten-membered rings.

The conformation of the eight-membered macrocycles in $\mathbf{1}$ and $\mathbf{1} \boldsymbol{a}$ was recognised by PLATON (Spek, 2009) as being closest to the TBC form (twist-boat chair; Evans \& Boeyens, 1989; Wiberg, 2003), which is consistent with $C_{2}$ point symmetry. Fig. 3 shows the overlay of the two structures based on the best fit of the PSSP fragment. The conformation of 2 was not assigned to any border type by PLATON, but Fig. 4 shows the puckering in $\mathbf{2}$ and $\mathbf{2} \boldsymbol{a}$ is distinctively different.

It is probably important to note that the intramolecular $\mathrm{C} 4-\mathrm{H} 4 B \cdots \mathrm{O} 1$ hydrogen bond (Table 2) stabilizes the tenmembered ring of $\mathbf{2}$.

\section{Supramolecular features}

The strongest intermolecular hydrogen-bonding interaction in $\mathbf{1}$ is between the anisyl ortho-hydrogen and macrocyclic O1 atoms and links the molecules into a diamondoid network.

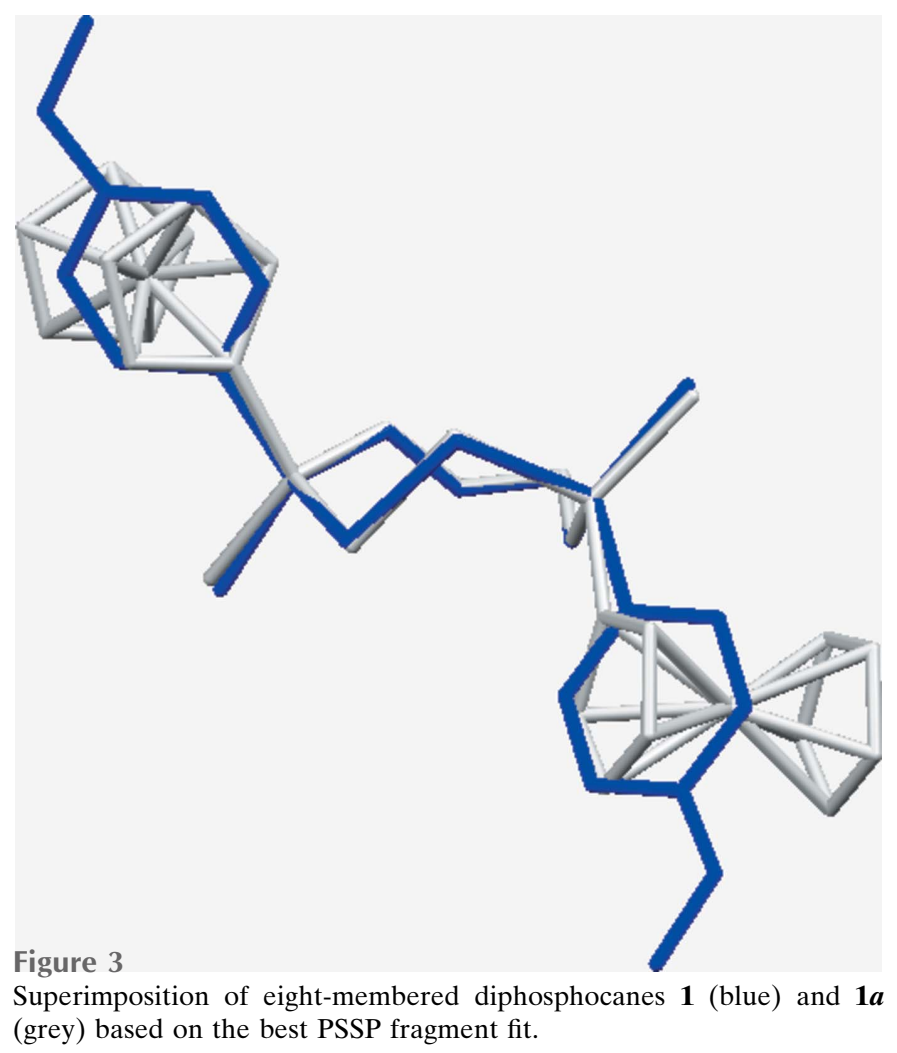

Table 2

Hydrogen-bond geometry $\left(\AA,^{\circ}\right)$ for $\mathbf{2}$.

$\mathrm{Cg}$ is the centroid of the $\mathrm{C} 20-\mathrm{C} 25$ ring.

\begin{tabular}{|c|c|c|c|c|}
\hline$D-\mathrm{H} \cdots A$ & $D-\mathrm{H}$ & $\mathrm{H} \cdots A$ & $D \cdots A$ & $D-\mathrm{H} \cdots A$ \\
\hline $\mathrm{C} 1-\mathrm{H} 1 B \cdots \mathrm{S} 3$ & 0.99 & 2.81 & $3.3883(2)$ & 118 \\
\hline $\mathrm{C} 4-\mathrm{H} 4 B \cdots \mathrm{O} 1$ & 0.99 & 2.48 & $3.1308(2)$ & 123 \\
\hline $\mathrm{C} 4-\mathrm{H} 4 B \cdots \mathrm{O} 4^{\mathrm{i}}$ & 0.99 & 2.56 & $3.2708(2)$ & 128 \\
\hline $\mathrm{C} 11-\mathrm{H} 11 \cdots \mathrm{O} 4^{\mathrm{i}}$ & 0.95 & 2.62 & $3.4951(3)$ & 154 \\
\hline $\mathrm{C} 24-\mathrm{H} 24 \cdots \mathrm{O}^{\mathrm{ii}}$ & 0.95 & 2.51 & $3.4240(3)$ & 162 \\
\hline $\mathrm{C} 16-\mathrm{H} 16 A \cdots C g^{\mathrm{iii}}$ & 0.98 & 2.62 & $3.454(8)$ & 143 \\
\hline
\end{tabular}

Symmetry codes:

(i) $\quad x,-y+\frac{1}{2}, z+\frac{1}{2}$

(ii) $\quad-x+1, y-\frac{1}{2},-z+\frac{1}{2}$;

$x-1,-y+\frac{1}{2}, z+\frac{1}{2}$.

There are no ring-stacking interactions since the shortest centroid-centroid distance is 5.0965 (3) A. The anisyl substituents may have inhibited this kind of interaction.

Intermolecular interactions in $\mathbf{2}$ are mainly based on the anisyl methoxyl $\mathrm{CH}_{3} \mathrm{O}$ oxygen atoms $\mathrm{O} 3$ and $\mathrm{O} 4$ and the $\mathrm{P}=\mathrm{S}$ sulfur atom S3 as acceptors. Hydrogen-bond donors are the anisyl ortho-hydrogen atoms or methylene hydrogen atoms. Moreover, some $\mathrm{C}-\mathrm{H}$.. $\pi$. interactions may play some role in the system, e.g. $\mathrm{C} 16-\mathrm{H} 16 A \cdots \operatorname{ring}(\mathrm{C} 20-\mathrm{C} 25)$, see Fig. 5. Again, the stacking interactions are weak since the closest intercentroid distance is equal to 4.9213 (4) $\AA$.

\section{Database survey}

Bisphosphonothioyl disulfanes represent a rather rare class of compounds (CSD Version 5.28, updated to Nov. 2016; Groom et al., 2016). Only three structures of cyclic bisphosphonothioyl disulfanes can be found in the database, HUGXAK, HUXEO and HUGXIS (ferrocenyl derivatives; Pillay et al., 2015) and four more will be available there soon (Hua et al., 2017). For structures of acyclic bisphosphonothioyl disulfanes

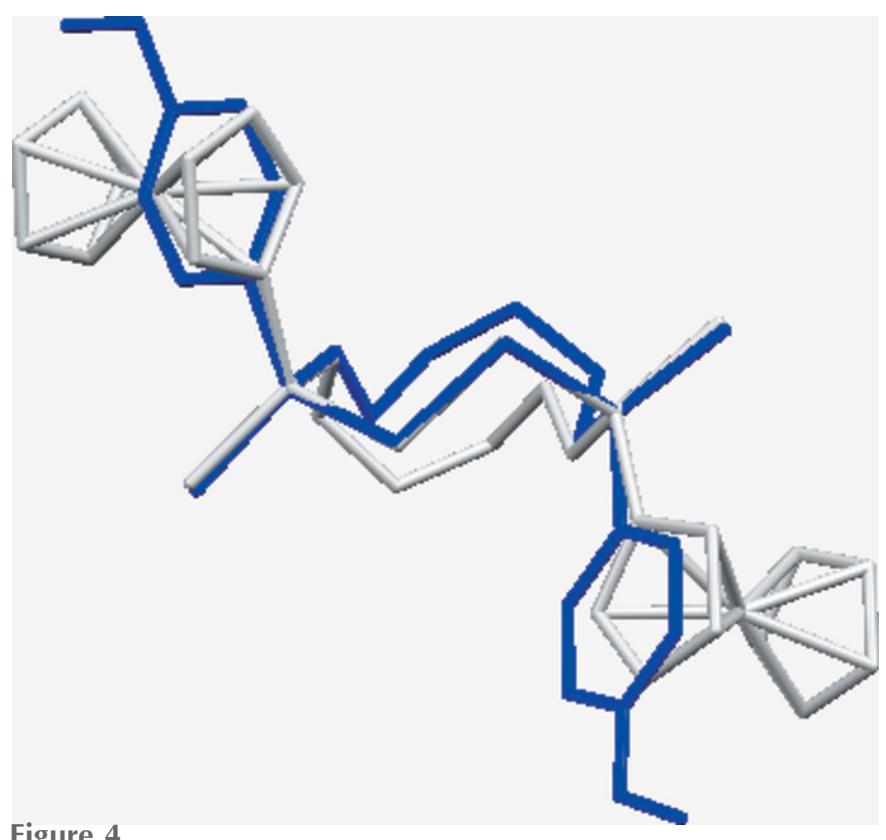

Figure 4

Overlay of ten-membered diphosphecanes $\mathbf{2}$ (blue) and $\mathbf{2 a}$ (grey) based on the best PSSP fragment fit. 
Table 3

Experimental details.

1

Crystal data

Chemical formula

$M_{\text {r }}$

Crystal system, space group

Temperature $(\mathrm{K})$

$a, b, c(\AA)$

$\alpha, \beta, \gamma\left({ }^{\circ}\right)$

$V\left(\AA^{3}\right)$

$Z$

Radiation type

$\mu\left(\mathrm{mm}^{-1}\right)$

Crystal size (mm)

Data collection

Diffractometer

Absorption correction

$T_{\text {min }}, T_{\text {max }}$

No. of measured, independent and observed $[I>$ $2 \sigma(I)]$ reflections

$R_{\text {int }}$

$(\sin \theta / \lambda)_{\max }\left(\AA^{-1}\right)$

Refinement

$R\left[F^{2}>2 \sigma\left(F^{2}\right)\right], w R\left(F^{2}\right), S$

No. of reflections

No. of parameters

$\mathrm{H}$-atom treatment

$\Delta \rho_{\max }, \Delta \rho_{\min }\left(\mathrm{e} \AA^{-3}\right)$

Absolute structure

Absolute structure parameter
2

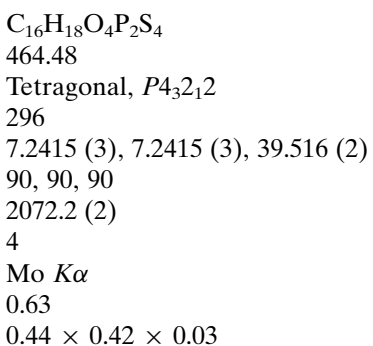

$\mathrm{C}_{18} \mathrm{H}_{22} \mathrm{O}_{4} \mathrm{P}_{2} \mathrm{~S}_{4}$

492.53

Monoclinic, $P 2_{1} / c$

120

9.4262 (6), $13.3761(8), 17.7998$ (13)

$90,90.068(7), 90$

2244.3 (3)

4

Mo $K \alpha$

0.59

$0.21 \times 0.20 \times 0.14$

Oxford Diffraction KM-4 CCD

Multi-scan (CrysAlis PRO; Agilent, 2011)

Oxford Diffraction KM-4 CCD

Analytical [CrysAlis PRO (Agilent, 2011) based on expressions derived by Clark \& Reid (1995)]

$0.689,0.98$

14211, 2019, 1839

$0.893,0.929$

9496, 4047, 3309

0.036

0.617

0.051

0.606

$0.038,0.092,1.09$

2019

120

$\mathrm{H}$-atom parameters constrained

$0.33,-0.22$

Refined as an inversion twin

0.45 (17)

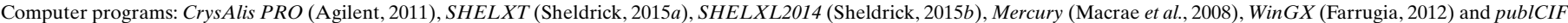
(Westrip, 2010).

see: FATTEA, FATTIE, FATVEC (Gray et al., 2004), YESDIY (Łopusiński et al., 1991), SIZHUF (Przychodzeń \& Chojnacki, 2008) and WAYMEO (Knopik et al., 1993).

\section{Synthesis and crystallization}

Eight- and ten-membered cyclic bisanisylphosphonothioyl disulfanes $\mathbf{1}$ and $\mathbf{2}$ were prepared using previously reported procedure (Przychodzeń, 2004). Compound 1 was fully spectroscopically characterized in that paper. Disulfane $\mathbf{2}$ is quite new, so all available spectroscopic data are given below. Both $\mathbf{1}$

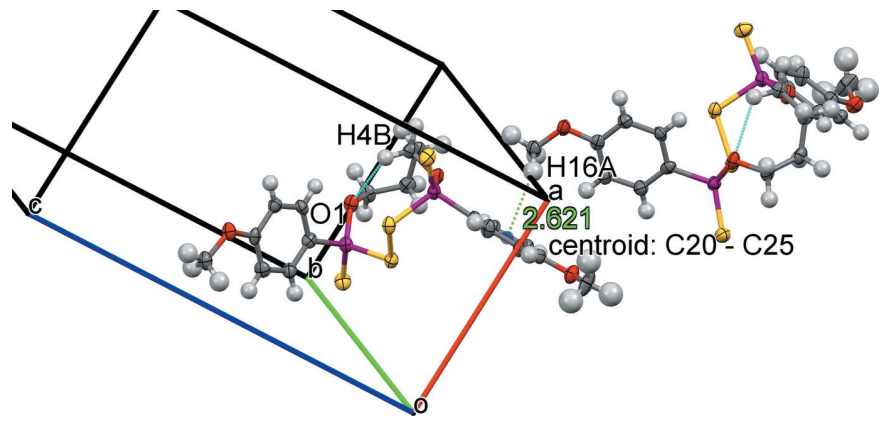

Figure 5

$\mathrm{C}-\mathrm{H} \cdots \pi$ interaction and internal $\mathrm{C}-\mathrm{H} \cdots \mathrm{O}$ hydrogen bonding in the ten-membered ring of $\mathbf{2}$. and $\mathbf{2}$ gave good quality colourless crystals after crystallization from ethyl acetate-cyclohexane $(1: 2 v / v)$ solvent system.

2,5-Bis(4-methoxyphenyl)-1,6,3,4,2,5-dioxadithiadiphosphocane 2,5-dithione, 1

M.p. 441-443 K.

2,5-Bis(4-methoxyphenyl)-1,6,3,4,2,5-dioxadithiadiphosphecane 2,5-dithione, 2

Yield: 65\%, m.p. 415-417 K.

${ }^{1} \mathrm{H}$ NMR $\left(\mathrm{CDCl}_{3}\right): 2.20\left(m, 2 \mathrm{H}, \mathrm{OCH}_{2} \mathrm{CH}_{2}\right), 2.25(m, 2 \mathrm{H}$, $\left.\mathrm{OCH}_{2} \mathrm{CH}_{2}\right), 3.89\left(s, 6 \mathrm{H}, \mathrm{OCH}_{3}\right), 4.37\left(d d d d,{ }^{3} J_{\mathrm{HH}}=11.6 \mathrm{~Hz}\right.$, ${ }^{2} J_{\mathrm{HH}}=10.4 \mathrm{~Hz},{ }^{3} J_{\mathrm{HP}}=5.4 \mathrm{~Hz},{ }^{3} J_{\mathrm{HH}}=2.2 \mathrm{~Hz}, 2 \mathrm{H}, \mathrm{OCH}_{A} \mathrm{H}_{\mathrm{B}}$ ), $4.89\left(d d t,{ }^{2} J_{\mathrm{HH}}=10.4 \mathrm{~Hz},{ }^{3} J_{\mathrm{HP}}=9.3 \mathrm{~Hz},{ }^{3} J_{\mathrm{HH}}=3.5 \mathrm{~Hz}, 2 \mathrm{H}\right.$, $\left.\mathrm{OCH}_{\mathrm{A}} H_{B}\right), 7.01\left(d d,{ }^{3} J_{\mathrm{HH}}=8.8 \mathrm{~Hz},{ }^{4} J_{\mathrm{HP}}=3.9 \mathrm{~Hz}, 4 \mathrm{H}, \mathrm{H}_{\text {meta }}\right)$, $7.87\left(d d,{ }^{3} J_{\mathrm{HP}}=14.2 \mathrm{~Hz},{ }^{3} J_{\mathrm{HH}}=8.8 \mathrm{~Hz}, 4 \mathrm{H}, \mathrm{H}_{\text {ortho }}\right)$.

${ }^{13} \mathrm{C}$ NMR: $27.21(d, J=6.9 \mathrm{~Hz}), 55.46(s), 67.08(d, J=$ $6.3 \mathrm{~Hz}), 114.03(d, J=17.5 \mathrm{~Hz}), 125.41(d, J=134 \mathrm{~Hz}), 132.89$ $(d, J=14.5 \mathrm{~Hz}), 163.09(s)$.

${ }^{31} \mathrm{P}\left\{{ }^{1} \mathrm{H}\right\}$ NMR $\left(\mathrm{CDCl}_{3}\right): 89.19\left({ }^{3} J_{\mathrm{PP}}=4 \mathrm{~Hz}\right)$

MS calculated for $\mathrm{C}_{18} \mathrm{H}_{22} \mathrm{O}_{4} \mathrm{P}_{2} \mathrm{~S}_{4}$ : 492.0. Found: 492.9 $[M+\mathrm{H}]^{+}$.

\section{Refinement}

Crystal data, data collection and structure refinement details are summarized in Table 3. Structure $\mathbf{1}$ was refined as an 
inversion twin with contribution of the second domain equal to 0.45 (17). This explains the ambiguous Flack parameter and is not surprising since we started from achiral substrates. Structure 2 was refined as a two-component rotational twin with twin law: $\left\{\begin{array}{lllllllll}\overline{1} & 0 & 0, & 0 & \overline{1} & 0, & 0 & 0 & 1\end{array}\right\}$ and $\mathrm{BASF}=0.767(3)$. Relatively high residual electron-density peaks in 2 ( $Q 1-Q 3 \mathrm{ca}$ 2e $\left.\AA^{3}\right)$, which are close to sulfur atoms ( $0.58 \AA$ from S4, $0.49 \AA$ from S2, $0.49 \AA$ from S1), may stem from conformational flexibility of the ring. Note: the structure of $\mathbf{1}$ was determined at room temperature (due to a failure of our CryoStream unit) not at $120 \mathrm{~K}$ as for $\mathbf{2}$ but we believe it did not influence the qualitative conclusions drawn from the results.

\section{Acknowledgements}

This research did not receive any specific grant from funding agencies in the public, commercial, or not-for-profit sectors. Conflict of interests: none.

\section{References}

Agilent (2011). CrysAlis PRO. Agilent Technologies, Yarnton, England.

Clark, R. C. \& Reid, J. S. (1995). Acta Cryst. A51, 887-897.

Evans, D. G. \& Boeyens, J. C. A. (1989). Acta Cryst. B45, 581-590.

Farrugia, L. J. (2012). J. Appl. Cryst. 45, 849-854.
Foreman, M. R. St J., Slawin, A. M. Z. \& Woollins, J. D. (1996). J. Chem. Soc. Dalton Trans. pp. 3653-3657.

Gray, I. P., Slawin, A. M. Z. \& Woollins, J. D. (2004). New J. Chem. 28, 1383-1389.

Groom, C. R., Bruno, I. J., Lightfoot, M. P. \& Ward, S. C. (2016). Acta Cryst. B72, 171-179.

Hua, G., Davidson, K., Cordes, D. B., Du, J., Slavin, A. M. Z. \& Woollins, J. D. (2017). Molecules, 22, 1687-1700.

Jesberger, M., Davis, T. P. \& Barner, L. (2003). Synthesis, pp. 1929 1958.

Knopik, P., Łuczak, L., Potrzebowski, M. J., Michalski, J., Błaszczyk, J. \& Wieczorek, M. W. (1993). J. Chem. Soc. Dalton Trans. pp. 27492757.

Łopusiński, A., Łuczak, L., Michalski, J., Kozioł, A. E. \& Gdaniec, M. (1991). Chem. Commun. pp. 889-890.

Macrae, C. F., Bruno, I. J., Chisholm, J. A., Edgington, P. R., McCabe, P., Pidcock, E., Rodriguez-Monge, L., Taylor, R., van de Streek, J. \& Wood, P. A. (2008). J. Appl. Cryst. 41, 466-470.

Pillay, M. N., van der Walt, H., Staples, R. J. \& van Zyl, W. E. (2015). J. Organomet. Chem. 794, 33-39.

Przychodzeń, W. (2004). Phosphorus Sulfur Silicon, 179, 1621-1633.

Przychodzeń, W. \& Chojnacki, J. (2008). Heteroat. Chem. 19, 271-282.

Sheldrick, G. M. (2015a). Acta Cryst. A71, 3-8.

Sheldrick, G. M. (2015b). Acta Cryst. C71, 3-8.

Spek, A. L. (2009). Acta Cryst. D65, 148-155.

Westrip, S. P. (2010). J. Appl. Cryst. 43, 920-925.

Wiberg, K. B. (2003). J. Org. Chem. 68, 9322-9329.

Zyl, W. E. van \& Woollins, J. D. (2013). Coord. Chem. Rev. 257, 718731. 


\section{supporting information}

Acta Cryst. (2018). E74, 212-216 [https://doi.org/10.1107/S2056989018001068]

\section{Crystal structures of eight- and ten-membered cyclic bisanisylphosphonothioyl disulfanes and comparison with their $\boldsymbol{P}$-ferrocenyl analogues}

\section{Witold Przychodzeń and Jaroslaw Chojnacki}

Computing details

For both structures, data collection: CrysAlis PRO (Agilent, 2011); cell refinement: CrysAlis PRO (Agilent, 2011); data reduction: CrysAlis PRO (Agilent, 2011); program(s) used to solve structure: SHELXT (Sheldrick, 2015a); program(s) used to refine structure: SHELXL2014 (Sheldrick, 2015b); molecular graphics: Mercury (Macrae et al., 2008). Software used to prepare material for publication: $\operatorname{Win} G X$ (Farrugia, 2012) and publCIF (Westrip, 2010) for (1); WinGX (Farrugia, 2012) for (2).

2,5-Bis(4-methoxyphenyl)-1,6,3,4,2 $\lambda^{5}, 5 \lambda^{5}$-dioxadithiadiphosphocane-2,5-dithione (1)

Crystal data

$\mathrm{C}_{16} \mathrm{H}_{18} \mathrm{O}_{4} \mathrm{P}_{2} \mathrm{~S}_{4}$

$M_{r}=464.48$

Tetragonal, $P 4_{3} 2_{1} 2$

Hall symbol: P 4nw 2abw

$a=7.2415(3) \AA$

$c=39.516(2) \AA$

$V=2072.2(2) \AA^{3}$

$Z=4$

$F(000)=960$

Data collection

Oxford Diffraction KM-4 CCD diffractometer

Detector resolution: 8.1883 pixels $\mathrm{mm}^{-1}$

$\omega$ scans, 0.40 deg width

Absorption correction: multi-scan

(CrysAlis PRO; Agilent, 2011)

$T_{\min }=0.689, T_{\max }=0.98$

14211 measured reflections

Refinement

Refinement on $F^{2}$

Least-squares matrix: full

$R\left[F^{2}>2 \sigma\left(F^{2}\right)\right]=0.038$

$w R\left(F^{2}\right)=0.092$

$S=1.09$

2019 reflections

120 parameters

0 restraints
$D_{\mathrm{x}}=1.489 \mathrm{Mg} \mathrm{m}^{-3}$

Mo $K \alpha$ radiation, $\lambda=0.71073 \AA$

Cell parameters from 6759 reflections

$\theta=2.1-32.4^{\circ}$

$\mu=0.63 \mathrm{~mm}^{-1}$

$T=296 \mathrm{~K}$

Plate, colourless

$0.44 \times 0.42 \times 0.03 \mathrm{~mm}$

2019 independent reflections

1839 reflections with $I>2 \sigma(I)$

$R_{\text {int }}=0.036$

$\theta_{\max }=26.0^{\circ}, \theta_{\min }=2.9^{\circ}$

$h=-8 \rightarrow 8$

$k=-8 \rightarrow 8$

$l=-48 \rightarrow 38$

Primary atom site location: structure-invariant direct methods

Secondary atom site location: difference Fourier map

Hydrogen site location: inferred from neighbouring sites

$\mathrm{H}$-atom parameters constrained

$w=1 /\left[\sigma^{2}\left(F_{\mathrm{o}}^{2}\right)+(0.0514 P)^{2}+0.5163 P\right]$

where $P=\left(F_{\mathrm{o}}^{2}+2 F_{\mathrm{c}}^{2}\right) / 3$ 
$(\Delta / \sigma)_{\max }<0.001$

$\Delta \rho_{\max }=0.33 \mathrm{e} \AA^{-3}$

$\Delta \rho_{\min }=-0.22$ e $\AA^{-3}$
Absolute structure: Refined as an inversion twin

Absolute structure parameter: 0.45 (17)

\section{Special details}

Geometry. All esds (except the esd in the dihedral angle between two 1.s. planes) are estimated using the full covariance matrix. The cell esds are taken into account individually in the estimation of esds in distances, angles and torsion angles; correlations between esds in cell parameters are only used when they are defined by crystal symmetry. An approximate (isotropic) treatment of cell esds is used for estimating esds involving l.s. planes.

Refinement. Refined as a 2-component inversion twin.

Fractional atomic coordinates and isotropic or equivalent isotropic displacement parameters $\left(\AA^{2}\right)$

\begin{tabular}{|c|c|c|c|c|}
\hline & $x$ & $y$ & $z$ & $U_{\text {iso }} * / U_{\text {eq }}$ \\
\hline $\mathrm{P} 1$ & $0.93887(12)$ & $0.21832(13)$ & $0.04117(2)$ & $0.0424(2)$ \\
\hline S1 & $0.84270(13)$ & $0.04364(17)$ & $0.00256(2)$ & $0.0584(3)$ \\
\hline S2 & 0.79007 (17) & $0.43697(17)$ & $0.03709(3)$ & $0.0715(4)$ \\
\hline $\mathrm{O} 1$ & $1.1556(3)$ & $0.2344(3)$ & $0.03669(5)$ & $0.0424(6)$ \\
\hline $\mathrm{O} 2$ & $0.8974(4)$ & $-0.1523(4)$ & $0.17469(6)$ & $0.0552(7)$ \\
\hline $\mathrm{C} 1$ & $1.2424(5)$ & $0.3553(5)$ & $0.01202(8)$ & $0.0450(8)$ \\
\hline $\mathrm{H} 1 \mathrm{~A}$ & 1.3209 & 0.4439 & 0.0235 & $0.054 *$ \\
\hline H1B & 1.1485 & 0.4228 & -0.0004 & $0.054 *$ \\
\hline $\mathrm{C} 10$ & $0.9277(5)$ & $0.1007(5)$ & $0.08087(8)$ & $0.0412(7)$ \\
\hline $\mathrm{C} 11$ & $1.0238(5)$ & $-0.0610(6)$ & $0.08670(9)$ & $0.0576(10)$ \\
\hline H11 & 1.0949 & -0.1113 & 0.0694 & $0.069^{*}$ \\
\hline $\mathrm{C} 12$ & $1.0170(6)$ & $-0.1496(6)$ & $0.11749(9)$ & $0.0584(10)$ \\
\hline H12 & 1.082 & -0.2589 & 0.1207 & $0.07 *$ \\
\hline $\mathrm{C} 13$ & $0.9144(5)$ & $-0.0768(5)$ & $0.14352(7)$ & $0.0434(8)$ \\
\hline C14 & $0.8206(6)$ & $0.0864(6)$ & $0.13813(9)$ & $0.0555(10)$ \\
\hline H14 & 0.7529 & 0.1384 & 0.1557 & $0.067^{*}$ \\
\hline $\mathrm{C} 15$ & $0.8252(6)$ & $0.1736(5)$ & $0.10720(9)$ & $0.0526(9)$ \\
\hline H15 & 0.759 & 0.2823 & 0.104 & $0.063 *$ \\
\hline $\mathrm{C} 16$ & $1.0028(6)$ & $-0.3130(6)$ & $0.18232(11)$ & $0.0672(12)$ \\
\hline H16A & 0.9613 & -0.4138 & 0.1685 & $0.101^{*}$ \\
\hline H16B & 0.9871 & -0.3442 & 0.2058 & $0.101^{*}$ \\
\hline H16C & 1.1309 & -0.2894 & 0.1779 & $0.101^{*}$ \\
\hline
\end{tabular}

Atomic displacement parameters $\left(\AA^{2}\right)$

\begin{tabular}{lllllll}
\hline & $U^{11}$ & $U^{22}$ & $U^{33}$ & $U^{12}$ & $U^{13}$ & $U^{23}$ \\
\hline P1 & $0.0373(4)$ & $0.0559(5)$ & $0.0340(4)$ & $0.0025(4)$ & $0.0018(3)$ & $0.0025(4)$ \\
S1 & $0.0423(5)$ & $0.0932(8)$ & $0.0396(5)$ & $-0.0164(5)$ & $-0.0034(4)$ & $-0.0029(5)$ \\
S2 & $0.0711(7)$ & $0.0755(7)$ & $0.0678(7)$ & $0.0285(6)$ & $0.0106(5)$ & $0.0156(6)$ \\
O1 & $0.0403(12)$ & $0.0552(14)$ & $0.0318(11)$ & $-0.0057(11)$ & $-0.0003(9)$ & $0.0010(10)$ \\
O2 & $0.0598(16)$ & $0.0694(17)$ & $0.0363(13)$ & $0.0070(13)$ & $0.0086(11)$ & $0.0069(11)$ \\
C1 & $0.054(2)$ & $0.0427(18)$ & $0.0381(17)$ & $-0.0144(16)$ & $0.0004(15)$ & $-0.0036(14)$ \\
C10 & $0.0358(16)$ & $0.054(2)$ & $0.0336(15)$ & $0.0004(15)$ & $0.0034(14)$ & $0.0006(14)$ \\
C11 & $0.056(2)$ & $0.076(3)$ & $0.0400(18)$ & $0.026(2)$ & $0.0145(16)$ & $0.0035(18)$ \\
C12 & $0.062(2)$ & $0.069(2)$ & $0.0443(19)$ & $0.0260(19)$ & $0.0073(17)$ & $0.0058(17)$
\end{tabular}




\begin{tabular}{lllllll}
$\mathrm{C} 13$ & $0.0363(18)$ & $0.061(2)$ & $0.0329(15)$ & $-0.0035(15)$ & $0.0030(13)$ & $0.0010(15)$ \\
$\mathrm{C} 14$ & $0.066(3)$ & $0.061(2)$ & $0.0402(18)$ & $0.0157(19)$ & $0.0169(16)$ & $-0.0040(17)$ \\
$\mathrm{C} 15$ & $0.065(3)$ & $0.050(2)$ & $0.0424(19)$ & $0.0145(18)$ & $0.0131(17)$ & $-0.0021(16)$ \\
$\mathrm{C} 16$ & $0.073(3)$ & $0.078(3)$ & $0.051(2)$ & $0.011(2)$ & $0.0064(19)$ & $0.016(2)$ \\
\hline
\end{tabular}

Geometric parameters $\left(\hat{A},{ }^{\circ}\right)$

\begin{tabular}{|c|c|c|c|}
\hline $\mathrm{P} 1-\mathrm{O} 1$ & $1.584(2)$ & $\mathrm{C} 10-\mathrm{C} 15$ & $1.383(5)$ \\
\hline $\mathrm{P} 1-\mathrm{C} 10$ & $1.787(3)$ & $\mathrm{C} 11-\mathrm{C} 12$ & $1.376(5)$ \\
\hline $\mathrm{P} 1-\mathrm{S} 2$ & $1.9220(14)$ & $\mathrm{C} 11-\mathrm{H} 11$ & 0.93 \\
\hline $\mathrm{P} 1-\mathrm{S} 1$ & $2.1006(13)$ & $\mathrm{C} 12-\mathrm{C} 13$ & $1.374(5)$ \\
\hline $\mathrm{S} 1-\mathrm{S} 1^{\mathrm{i}}$ & $2.068(2)$ & $\mathrm{C} 12-\mathrm{H} 12$ & 0.93 \\
\hline $\mathrm{O} 1-\mathrm{C} 1$ & $1.453(4)$ & $\mathrm{C} 13-\mathrm{C} 14$ & $1.380(5)$ \\
\hline $\mathrm{O} 2-\mathrm{C} 13$ & $1.353(4)$ & $\mathrm{C} 14-\mathrm{C} 15$ & $1.376(5)$ \\
\hline $\mathrm{O} 2-\mathrm{C} 16$ & $1.424(5)$ & $\mathrm{C} 14-\mathrm{H} 14$ & 0.93 \\
\hline $\mathrm{C} 1-\mathrm{C}^{\mathrm{i}}$ & $1.496(7)$ & $\mathrm{C} 15-\mathrm{H} 15$ & 0.93 \\
\hline $\mathrm{C} 1-\mathrm{H} 1 \mathrm{~A}$ & 0.97 & $\mathrm{C} 16-\mathrm{H} 16 \mathrm{~A}$ & 0.96 \\
\hline $\mathrm{C} 1-\mathrm{H} 1 \mathrm{~B}$ & 0.97 & $\mathrm{C} 16-\mathrm{H} 16 \mathrm{~B}$ & 0.96 \\
\hline $\mathrm{C} 10-\mathrm{C} 11$ & $1.382(5)$ & $\mathrm{C} 16-\mathrm{H} 16 \mathrm{C}$ & 0.96 \\
\hline $\mathrm{O} 1-\mathrm{P} 1-\mathrm{C} 10$ & $100.26(14)$ & $\mathrm{C} 10-\mathrm{C} 11-\mathrm{H} 11$ & 119.2 \\
\hline $\mathrm{O} 1-\mathrm{P} 1-\mathrm{S} 2$ & $119.05(11)$ & $\mathrm{C} 13-\mathrm{C} 12-\mathrm{C} 11$ & $120.2(4)$ \\
\hline $\mathrm{C} 10-\mathrm{P} 1-\mathrm{S} 2$ & $116.15(13)$ & $\mathrm{C} 13-\mathrm{C} 12-\mathrm{H} 12$ & 119.9 \\
\hline $\mathrm{O} 1-\mathrm{P} 1-\mathrm{S} 1$ & $106.97(9)$ & $\mathrm{C} 11-\mathrm{C} 12-\mathrm{H} 12$ & 119.9 \\
\hline $\mathrm{C} 10-\mathrm{P} 1-\mathrm{S} 1$ & $109.61(12)$ & $\mathrm{O} 2-\mathrm{C} 13-\mathrm{C} 12$ & $125.1(3)$ \\
\hline $\mathrm{S} 2-\mathrm{P} 1-\mathrm{S} 1$ & $104.44(6)$ & $\mathrm{O} 2-\mathrm{C} 13-\mathrm{C} 14$ & $116.2(3)$ \\
\hline $\mathrm{S} 1-\mathrm{S} 1-\mathrm{P} 1$ & $105.17(6)$ & $\mathrm{C} 12-\mathrm{C} 13-\mathrm{C} 14$ & $118.6(3)$ \\
\hline $\mathrm{C} 1-\mathrm{O} 1-\mathrm{P} 1$ & $123.2(2)$ & $\mathrm{C} 15-\mathrm{C} 14-\mathrm{C} 13$ & $121.2(3)$ \\
\hline $\mathrm{C} 13-\mathrm{O} 2-\mathrm{C} 16$ & $118.3(3)$ & $\mathrm{C} 15-\mathrm{C} 14-\mathrm{H} 14$ & 119.4 \\
\hline $\mathrm{O} 1-\mathrm{C} 1-\mathrm{C}^{\mathrm{i}}$ & $109.5(3)$ & $\mathrm{C} 13-\mathrm{C} 14-\mathrm{H} 14$ & 119.4 \\
\hline $\mathrm{O} 1-\mathrm{C} 1-\mathrm{H} 1 \mathrm{~A}$ & 109.8 & $\mathrm{C} 14-\mathrm{C} 15-\mathrm{C} 10$ & $120.4(3)$ \\
\hline $\mathrm{C} 1{ }^{\mathrm{i}}-\mathrm{C} 1-\mathrm{H} 1 \mathrm{~A}$ & 109.8 & $\mathrm{C} 14-\mathrm{C} 15-\mathrm{H} 15$ & 119.8 \\
\hline $\mathrm{O} 1-\mathrm{C} 1-\mathrm{H} 1 \mathrm{~B}$ & 109.8 & $\mathrm{C} 10-\mathrm{C} 15-\mathrm{H} 15$ & 119.8 \\
\hline $\mathrm{C} 1{ }^{\mathrm{i}}-\mathrm{C} 1-\mathrm{H} 1 \mathrm{~B}$ & 109.8 & $\mathrm{O} 2-\mathrm{C} 16-\mathrm{H} 16 \mathrm{~A}$ & 109.5 \\
\hline $\mathrm{H} 1 \mathrm{~A}-\mathrm{C} 1-\mathrm{H} 1 \mathrm{~B}$ & 108.2 & $\mathrm{O} 2-\mathrm{C} 16-\mathrm{H} 16 \mathrm{~B}$ & 109.5 \\
\hline $\mathrm{C} 11-\mathrm{C} 10-\mathrm{C} 15$ & $117.9(3)$ & $\mathrm{H} 16 \mathrm{~A}-\mathrm{C} 16-\mathrm{H} 16 \mathrm{~B}$ & 109.5 \\
\hline $\mathrm{C} 11-\mathrm{C} 10-\mathrm{P} 1$ & $121.8(3)$ & $\mathrm{O} 2-\mathrm{C} 16-\mathrm{H} 16 \mathrm{C}$ & 109.5 \\
\hline $\mathrm{C} 15-\mathrm{C} 10-\mathrm{P} 1$ & $120.2(3)$ & $\mathrm{H} 16 \mathrm{~A}-\mathrm{C} 16-\mathrm{H} 16 \mathrm{C}$ & 109.5 \\
\hline $\mathrm{C} 12-\mathrm{C} 11-\mathrm{C} 10$ & $121.6(3)$ & $\mathrm{H} 16 \mathrm{~B}-\mathrm{C} 16-\mathrm{H} 16 \mathrm{C}$ & 109.5 \\
\hline $\mathrm{C} 12-\mathrm{C} 11-\mathrm{H} 11$ & 119.2 & & \\
\hline $\mathrm{C} 10-\mathrm{P} 1-\mathrm{O} 1-\mathrm{C} 1$ & $-165.8(2)$ & $\mathrm{P} 1-\mathrm{C} 10-\mathrm{C} 11-\mathrm{C} 12$ & $179.0(3)$ \\
\hline $\mathrm{S} 2-\mathrm{P} 1-\mathrm{O} 1-\mathrm{C} 1$ & $-38.0(3)$ & $\mathrm{C} 10-\mathrm{C} 11-\mathrm{C} 12-\mathrm{C} 13$ & $-0.6(7)$ \\
\hline $\mathrm{S} 1-\mathrm{P} 1-\mathrm{O} 1-\mathrm{C} 1$ & $79.8(2)$ & $\mathrm{C} 16-\mathrm{O} 2-\mathrm{C} 13-\mathrm{C} 12$ & $4.4(6)$ \\
\hline $\mathrm{P} 1-\mathrm{O} 1-\mathrm{C} 1-\mathrm{C}^{\mathrm{i}}$ & $-119.6(3)$ & $\mathrm{C} 16-\mathrm{O} 2-\mathrm{C} 13-\mathrm{C} 14$ & $-175.3(4)$ \\
\hline $\mathrm{O} 1-\mathrm{P} 1-\mathrm{C} 10-\mathrm{C} 11$ & $-52.2(3)$ & $\mathrm{C} 11-\mathrm{C} 12-\mathrm{C} 13-\mathrm{O} 2$ & $179.8(4)$ \\
\hline $\mathrm{S} 2-\mathrm{P} 1-\mathrm{C} 10-\mathrm{C} 11$ & $178.1(3)$ & $\mathrm{C} 11-\mathrm{C} 12-\mathrm{C} 13-\mathrm{C} 14$ & $-0.5(6)$ \\
\hline $\mathrm{S} 1-\mathrm{P} 1-\mathrm{C} 10-\mathrm{C} 11$ & $60.1(3)$ & $\mathrm{O} 2-\mathrm{C} 13-\mathrm{C} 14-\mathrm{C} 15$ & $-178.8(4)$ \\
\hline
\end{tabular}




$\begin{array}{llll}\mathrm{O} 1-\mathrm{P} 1-\mathrm{C} 10-\mathrm{C} 15 & 125.9(3) & \mathrm{C} 12-\mathrm{C} 13-\mathrm{C} 14-\mathrm{C} 15 & 1.5(6) \\ \mathrm{S} 2-\mathrm{P} 1-\mathrm{C} 10-\mathrm{C} 15 & -3.8(4) & \mathrm{C} 13-\mathrm{C} 14-\mathrm{C} 15-\mathrm{C} 10 & -1.2(7) \\ \mathrm{S} 1-\mathrm{P} 1-\mathrm{C} 10-\mathrm{C} 15 & -121.9(3) & \mathrm{C} 11-\mathrm{C} 10-\mathrm{C} 15-\mathrm{C} 14 & 0.1(6) \\ \mathrm{C} 15-\mathrm{C} 10-\mathrm{C} 11-\mathrm{C} 12 & 0.9(6) & \mathrm{P} 1-\mathrm{C} 10-\mathrm{C} 15-\mathrm{C} 14 & -178.1(3)\end{array}$

Symmetry code: (i) $y+1, x-1,-z$.

Hydrogen-bond geometry $\left(A,{ }^{\circ}\right)$

\begin{tabular}{lllll}
\hline$D-\mathrm{H} \cdots A$ & $D-\mathrm{H}$ & $\mathrm{H} \cdots A$ & $D \cdots A$ & $D-\mathrm{H} \cdots A$ \\
\hline $\mathrm{C} 1-\mathrm{H} 1 A \cdots \mathrm{O} 2^{\mathrm{ii}}$ & 0.97 & 2.60 & $3.4843(2)$ & 151 \\
$\mathrm{C} 14-\mathrm{H} 14 \cdots \mathrm{O} 1^{\mathrm{iii}}$ & 0.93 & 2.55 & $3.4548(2)$ & 163 \\
\hline
\end{tabular}

Symmetry codes: (ii) $x+1 / 2,-y+1 / 2,-z+1 / 4$; (iii) $x-1 / 2,-y+1 / 2,-z+1 / 4$.

2,5-Bis(4-methoxyphenyl)-1,6,3,4,2 $\lambda^{5}, 5 \lambda^{5}$-dioxadithiadiphosphecane-2,5-dithione (2)

\section{Crystal data}

$\mathrm{C}_{18} \mathrm{H}_{22} \mathrm{O}_{4} \mathrm{P}_{2} \mathrm{~S}_{4}$

$M_{r}=492.53$

Monoclinic, $P 2_{1} / c$

Hall symbol: -P $2 \mathrm{ybc}$

$a=9.4262(6) \AA$

$b=13.3761(8) \AA$

$c=17.7998(13) \AA$

$\beta=90.068(7)^{\circ}$

$V=2244.3(3) \AA^{3}$

$Z=4$

\section{Data collection}

\section{Oxford Diffraction KM-4 CCD}

diffractometer

Graphite monochromator

Detector resolution: 8.19 pixels $\mathrm{mm}^{-1}$

$\omega$ scans

Absorption correction: analytical

[CrysAlis PRO (Agilent, 2011) based on

expressions derived by Clark \& Reid (1995)]

$T_{\min }=0.893, T_{\max }=0.929$

\section{Refinement}

Refinement on $F^{2}$

Least-squares matrix: full

$R\left[F^{2}>2 \sigma\left(F^{2}\right)\right]=0.082$

$w R\left(F^{2}\right)=0.241$

$S=1.05$

4047 reflections

256 parameters

0 restraints

Primary atom site location: structure-invariant direct methods
$F(000)=1024$

$D_{\mathrm{x}}=1.458 \mathrm{Mg} \mathrm{m}^{-3}$

Mo $K \alpha$ radiation, $\lambda=0.71073 \AA$

Cell parameters from 5521 reflections

$\theta=1.9-28.8^{\circ}$

$\mu=0.59 \mathrm{~mm}^{-1}$

$T=120 \mathrm{~K}$

Prism, colourless

$0.21 \times 0.20 \times 0.14 \mathrm{~mm}$

9496 measured reflections

4047 independent reflections

3309 reflections with $I>2 \sigma(I)$

$R_{\text {int }}=0.051$

$\theta_{\max }=25.5^{\circ}, \theta_{\min }=1.9^{\circ}$

$h=-6 \rightarrow 11$

$k=-13 \rightarrow 16$

$l=-21 \rightarrow 18$

Secondary atom site location: difference Fourier map

Hydrogen site location: inferred from neighbouring sites

$\mathrm{H}$-atom parameters constrained

$w=1 /\left[\sigma^{2}\left(F_{\mathrm{o}}^{2}\right)+(0.1828 P)^{2}+0.8642 P\right]$

where $P=\left(F_{\mathrm{o}}{ }^{2}+2 F_{\mathrm{c}}{ }^{2}\right) / 3$

$(\Delta / \sigma)_{\max }<0.001$

$\Delta \rho_{\max }=2.27 \mathrm{e} \AA^{-3}$

$\Delta \rho_{\min }=-0.84$ e $\AA^{-3}$ 


\section{Special details}

Geometry. All esds (except the esd in the dihedral angle between two 1.s. planes) are estimated using the full covariance matrix. The cell esds are taken into account individually in the estimation of esds in distances, angles and torsion angles; correlations between esds in cell parameters are only used when they are defined by crystal symmetry. An approximate (isotropic) treatment of cell esds is used for estimating esds involving l.s. planes.

Refinement. Refined as a 2-component twin.

Fractional atomic coordinates and isotropic or equivalent isotropic displacement parameters $\left(\hat{A}^{2}\right)$

\begin{tabular}{|c|c|c|c|c|}
\hline & $x$ & $y$ & $z$ & $U_{\text {iso }} * / U_{\text {eq }}$ \\
\hline $\mathrm{P} 1$ & 0.34560 (19) & $0.44281(13)$ & $0.17369(10)$ & $0.0294(4)$ \\
\hline $\mathrm{P} 2$ & 0.74857 (19) & $0.25294(12)$ & $0.13780(10)$ & $0.0288(4)$ \\
\hline $\mathrm{S} 1$ & 0.40975 (19) & $0.31382(12)$ & $0.11377(10)$ & $0.0318(4)$ \\
\hline $\mathrm{S} 2$ & $0.5483(2)$ & $0.24408(12)$ & $0.18767(10)$ & $0.0325(4)$ \\
\hline $\mathrm{S} 3$ & 0.1939 (2) & $0.49814(14)$ & $0.11372(11)$ & $0.0384(5)$ \\
\hline S4 & $0.8724(2)$ & $0.19798(14)$ & $0.21394(12)$ & $0.0413(5)$ \\
\hline $\mathrm{O} 1$ & $0.4806(6)$ & $0.5097(3)$ & $0.1892(3)$ & $0.0342(11)$ \\
\hline $\mathrm{O} 2$ & $0.7721(6)$ & $0.3641(3)$ & $0.1101(3)$ & 0.0348 (11) \\
\hline $\mathrm{O} 3$ & $0.2227(5)$ & $0.2847(4)$ & $0.4774(3)$ & $0.0344(11)$ \\
\hline O4 & $0.7677(6)$ & $0.0419(4)$ & -0.1545 & 0.0377 (12) \\
\hline $\mathrm{C} 1$ & $0.5242(8)$ & $0.5920(5)$ & $0.1389(4)$ & $0.0356(16)$ \\
\hline $\mathrm{H} 1 \mathrm{~A}$ & 0.5618 & 0.6477 & 0.1696 & $0.043^{*}$ \\
\hline H1B & 0.4399 & 0.6169 & 0.1114 & $0.043^{*}$ \\
\hline $\mathrm{C} 2$ & $0.6350(8)$ & $0.5604(5)$ & $0.0833(4)$ & $0.0357(16)$ \\
\hline $\mathrm{H} 2 \mathrm{~A}$ & 0.6429 & 0.613 & 0.0444 & $0.043^{*}$ \\
\hline $\mathrm{H} 2 \mathrm{~B}$ & 0.6019 & 0.4987 & 0.0581 & $0.043^{*}$ \\
\hline $\mathrm{C} 3$ & $0.7811(9)$ & $0.5412(5)$ & $0.1152(5)$ & $0.0392(17)$ \\
\hline $\mathrm{H} 3 \mathrm{~A}$ & 0.8083 & 0.5991 & 0.1467 & $0.047^{*}$ \\
\hline H3B & 0.8493 & 0.5376 & 0.073 & $0.047^{*}$ \\
\hline $\mathrm{C} 4$ & $0.7962(8)$ & $0.4456(5)$ & $0.1626(4)$ & $0.0335(15)$ \\
\hline $\mathrm{H} 4 \mathrm{~A}$ & 0.8923 & 0.441 & 0.1849 & $0.04 *$ \\
\hline H4B & 0.7252 & 0.4443 & 0.2035 & $0.04 *$ \\
\hline $\mathrm{C} 10$ & $0.3054(7)$ & $0.3994(5)$ & $0.2668(4)$ & $0.0303(15)$ \\
\hline $\mathrm{C} 11$ & $0.4091(7)$ & $0.3973(5)$ & $0.3230(4)$ & $0.0288(14)$ \\
\hline H11 & 0.501 & 0.4233 & 0.3132 & $0.035^{*}$ \\
\hline $\mathrm{C} 12$ & $0.3784(7)$ & $0.3576(5)$ & 0.3929 (4) & $0.0311(15)$ \\
\hline H12 & 0.4494 & 0.3549 & 0.4307 & $0.037^{*}$ \\
\hline $\mathrm{C} 13$ & $0.2422(8)$ & $0.3216(5)$ & $0.4072(4)$ & $0.0302(15)$ \\
\hline C14 & $0.1390(8)$ & $0.3237(5)$ & $0.3512(4)$ & $0.0343(16)$ \\
\hline H14 & 0.0465 & 0.2985 & 0.3607 & $0.041^{*}$ \\
\hline C15 & $0.1728(8)$ & $0.3631(5)$ & 0.2814 & $0.0341(15)$ \\
\hline H15 & 0.1026 & 0.3649 & 0.2431 & $0.041 *$ \\
\hline $\mathrm{C} 16$ & $0.0843(8)$ & 0.2559 & $0.4988(5)$ & 0.0377 (17) \\
\hline H16A & 0.0202 & 0.3133 & 0.4942 & $0.057^{*}$ \\
\hline H16B & 0.0854 & 0.2327 & 0.5511 & $0.057^{*}$ \\
\hline $\mathrm{H} 16 \mathrm{C}$ & 0.0513 & 0.2017 & 0.4661 & $0.057^{*}$ \\
\hline $\mathrm{C} 20$ & $0.7453(7)$ & 0.1893 & $0.0491(4)$ & $0.0291(14)$ \\
\hline $\mathrm{C} 21$ & $0.7321(8)$ & $0.2409(5)$ & $-0.0182(4)$ & $0.0302(15)$ \\
\hline
\end{tabular}


supporting information

\begin{tabular}{lllll}
$\mathrm{H} 21$ & 0.7173 & 0.3112 & -0.018 & $0.036^{*}$ \\
$\mathrm{C} 22$ & $0.7406(8)$ & $0.1894(5)$ & $-0.0860(4)$ & $0.0320(15)$ \\
$\mathrm{H} 22$ & 0.7333 & 0.2242 & -0.1323 & $0.038^{*}$ \\
$\mathrm{C} 23$ & $0.7602(7)$ & $0.0850(5)$ & $-0.0852(4)$ & $0.0302(14)$ \\
$\mathrm{C} 24$ & $0.7703(9)$ & $0.0347(5)$ & $-0.0185(4)$ & $0.0372(17)$ \\
$\mathrm{H} 24$ & 0.7819 & -0.0359 & -0.0183 & $0.045^{*}$ \\
$\mathrm{C} 25$ & $0.7636(9)$ & $0.0867(5)$ & $0.0488(4)$ & $0.0368(16)$ \\
$\mathrm{H} 25$ & 0.7716 & 0.0517 & 0.0951 & $0.044^{*}$ \\
$\mathrm{C} 26$ & $0.7930(13)$ & $-0.0639(6)$ & $-0.1555(5)$ & $0.058(3)$ \\
$\mathrm{H} 26 \mathrm{~A}$ & 0.8789 & -0.0789 & -0.1266 & $0.087^{*}$ \\
$\mathrm{H} 26 \mathrm{~B}$ & 0.8055 & -0.0863 & -0.2075 & $0.087^{*}$ \\
$\mathrm{H} 26 \mathrm{C}$ & 0.7119 & -0.0986 & -0.1331 & $0.087^{*}$ \\
\hline
\end{tabular}

Atomic displacement parameters $\left(\AA^{2}\right)$

\begin{tabular}{|c|c|c|c|c|c|c|}
\hline & $U^{11}$ & $U^{22}$ & $U^{33}$ & $U^{12}$ & $U^{13}$ & $U^{23}$ \\
\hline P1 & $0.0321(9)$ & $0.0271(9)$ & $0.0289(9)$ & $0.0023(7)$ & $0.0031(7)$ & $0.0019(7)$ \\
\hline P2 & $0.0358(9)$ & $0.0216(8)$ & $0.0292(10)$ & $0.0003(7)$ & $0.0061(8)$ & $-0.0005(6)$ \\
\hline S1 & $0.0380(9)$ & $0.0291(8)$ & $0.0285(9)$ & $-0.0009(7)$ & $0.0034(8)$ & $-0.0020(7)$ \\
\hline S2 & $0.0407(9)$ & $0.0271(8)$ & $0.0299(9)$ & $0.0032(7)$ & $0.0086(8)$ & $0.0039(7)$ \\
\hline S3 & $0.0399(9)$ & $0.0405(10)$ & $0.0348(10)$ & $0.0079(7)$ & $0.0008(8)$ & $0.0061(8)$ \\
\hline S4 & $0.0486(11)$ & $0.0347(10)$ & $0.0404(11)$ & $0.0097(8)$ & $-0.0055(9)$ & $-0.0018(8)$ \\
\hline $\mathrm{O} 1$ & $0.047(3)$ & $0.025(2)$ & $0.030(3)$ & $-0.001(2)$ & $0.007(2)$ & $0.005(2)$ \\
\hline $\mathrm{O} 2$ & $0.051(3)$ & $0.021(2)$ & $0.033(3)$ & $-0.005(2)$ & $0.005(2)$ & $-0.002(2)$ \\
\hline $\mathrm{O} 3$ & 0.040 & $0.031(2)$ & $0.032(3)$ & $0.004(2)$ & $0.008(2)$ & $0.007(2)$ \\
\hline $\mathrm{O} 4$ & $0.051(3)$ & 0.030 & $0.032(3)$ & $0.008(2)$ & $0.000(2)$ & -0.003 \\
\hline $\mathrm{C} 1$ & $0.043(4)$ & $0.021(3)$ & $0.043(4)$ & $-0.001(3)$ & 0.010 & 0.008 \\
\hline $\mathrm{C} 2$ & $0.047(4)$ & $0.026(3)$ & $0.034(4)$ & $-0.007(3)$ & $0.002(3)$ & $0.006(3)$ \\
\hline $\mathrm{C} 3$ & $0.052(4)$ & $0.023(3)$ & $0.042(4)$ & $-0.007(3)$ & $0.001(4)$ & $0.000(3)$ \\
\hline $\mathrm{C} 4$ & $0.040(4)$ & $0.025(3)$ & $0.035(4)$ & $-0.001(3)$ & $0.001(3)$ & $-0.002(3)$ \\
\hline $\mathrm{C} 10$ & $0.036(3)$ & $0.022(3)$ & $0.032(4)$ & $0.006(3)$ & $-0.003(3)$ & $0.001(3)$ \\
\hline $\mathrm{C} 11$ & $0.025(3)$ & $0.027(3)$ & $0.035(4)$ & $0.003(2)$ & 0.008 (3) & $0.000(3)$ \\
\hline $\mathrm{C} 12$ & $0.033(4)$ & $0.029(3)$ & $0.031(4)$ & 0.003 & $0.001(3)$ & -0.003 \\
\hline $\mathrm{C} 13$ & $0.040(4)$ & 0.020 & $0.030(4)$ & $0.008(3)$ & $0.007(3)$ & -0.003 \\
\hline $\mathrm{C} 14$ & $0.038(4)$ & $0.027(3)$ & $0.038(4)$ & $-0.004(3)$ & 0.005 & $0.004(3)$ \\
\hline $\mathrm{C} 15$ & $0.034(4)$ & $0.037(4)$ & $0.032(4)$ & $0.003(3)$ & $0.002(3)$ & -0.001 \\
\hline $\mathrm{C} 16$ & $0.042(4)$ & $0.033(4)$ & $0.038(4)$ & $0.006(3)$ & 0.010 & $0.001(3)$ \\
\hline $\mathrm{C} 20$ & $0.030(3)$ & $0.026(3)$ & $0.031(4)$ & $-0.002(3)$ & 0.009 (3) & $-0.002(3)$ \\
\hline $\mathrm{C} 21$ & $0.037(4)$ & $0.024(3)$ & $0.030(4)$ & $0.001(3)$ & $0.001(3)$ & $0.000(3)$ \\
\hline $\mathrm{C} 22$ & $0.035(4)$ & $0.028(3)$ & $0.033(4)$ & 0.000 & -0.001 & $0.001(3)$ \\
\hline $\mathrm{C} 23$ & 0.028 (3) & 0.029 (3) & $0.034(4)$ & $0.003(3)$ & $0.003(3)$ & $-0.005(3)$ \\
\hline $\mathrm{C} 24$ & $0.057(5)$ & 0.020 & $0.035(4)$ & $-0.005(3)$ & 0.009 (4) & -0.003 \\
\hline $\mathrm{C} 25$ & $0.051(4)$ & $0.029(4)$ & $0.031(4)$ & $-0.003(3)$ & $0.009(3)$ & $0.004(3)$ \\
\hline $\mathrm{C} 26$ & $0.106(8)$ & $0.030(4)$ & $0.037(5)$ & $0.024(5)$ & $0.000(5)$ & -0.010 \\
\hline
\end{tabular}


Geometric parameters $\left(\AA,{ }^{\circ}\right)$

\begin{tabular}{|c|c|c|c|}
\hline $\mathrm{P} 1-\mathrm{O} 1$ & $1.580(5)$ & $\mathrm{C} 10-\mathrm{C} 15$ & $1.366(10)$ \\
\hline $\mathrm{P} 1-\mathrm{C} 10$ & $1.798(7)$ & $\mathrm{C} 10-\mathrm{C} 11$ & $1.398(9)$ \\
\hline $\mathrm{P} 1-\mathrm{S} 3$ & $1.931(3)$ & $\mathrm{C} 11-\mathrm{C} 12$ & $1.383(10)$ \\
\hline $\mathrm{P} 1-\mathrm{S} 1$ & $2.117(2)$ & $\mathrm{C} 11-\mathrm{H} 11$ & 0.95 \\
\hline $\mathrm{P} 2-\mathrm{O} 2$ & $1.582(5)$ & $\mathrm{C} 12-\mathrm{C} 13$ & $1.395(10)$ \\
\hline $\mathrm{P} 2-\mathrm{C} 20$ & $1.794(7)$ & $\mathrm{C} 12-\mathrm{H} 12$ & 0.95 \\
\hline $\mathrm{P} 2-\mathrm{S} 4$ & $1.933(3)$ & $\mathrm{C} 13-\mathrm{C} 14$ & $1.393(10)$ \\
\hline $\mathrm{P} 2-\mathrm{S} 2$ & $2.091(3)$ & $\mathrm{C} 14-\mathrm{C} 15$ & $1.387(10)$ \\
\hline $\mathrm{S} 1-\mathrm{S} 2$ & $2.074(3)$ & $\mathrm{C} 14-\mathrm{H} 14$ & 0.95 \\
\hline $\mathrm{O} 1-\mathrm{C} 1$ & $1.477(8)$ & $\mathrm{C} 15-\mathrm{H} 15$ & 0.95 \\
\hline $\mathrm{O} 2-\mathrm{C} 4$ & $1.453(8)$ & C16-H16A & 0.98 \\
\hline $\mathrm{O} 3-\mathrm{C} 13$ & $1.357(8)$ & C16-H16B & 0.98 \\
\hline $\mathrm{O} 3-\mathrm{C} 16$ & $1.413(9)$ & $\mathrm{C} 16-\mathrm{H} 16 \mathrm{C}$ & 0.98 \\
\hline $\mathrm{O} 4-\mathrm{C} 23$ & $1.364(8)$ & $\mathrm{C} 20-\mathrm{C} 25$ & $1.383(10)$ \\
\hline $\mathrm{O} 4-\mathrm{C} 26$ & $1.435(9)$ & $\mathrm{C} 20-\mathrm{C} 21$ & $1.389(10)$ \\
\hline $\mathrm{C} 1-\mathrm{C} 2$ & $1.501(11)$ & $\mathrm{C} 21-\mathrm{C} 22$ & $1.391(10)$ \\
\hline $\mathrm{C} 1-\mathrm{H} 1 \mathrm{~A}$ & 0.99 & $\mathrm{C} 21-\mathrm{H} 21$ & 0.95 \\
\hline $\mathrm{C} 1-\mathrm{H} 1 \mathrm{~B}$ & 0.99 & $\mathrm{C} 22-\mathrm{C} 23$ & $1.408(10)$ \\
\hline $\mathrm{C} 2-\mathrm{C} 3$ & $1.511(11)$ & $\mathrm{C} 22-\mathrm{H} 22$ & 0.95 \\
\hline $\mathrm{C} 2-\mathrm{H} 2 \mathrm{~A}$ & 0.99 & $\mathrm{C} 23-\mathrm{C} 24$ & $1.368(10)$ \\
\hline $\mathrm{C} 2-\mathrm{H} 2 \mathrm{~B}$ & 0.99 & $\mathrm{C} 24-\mathrm{C} 25$ & $1.388(10)$ \\
\hline $\mathrm{C} 3-\mathrm{C} 4$ & $1.539(10)$ & $\mathrm{C} 24-\mathrm{H} 24$ & 0.95 \\
\hline $\mathrm{C} 3-\mathrm{H} 3 \mathrm{~A}$ & 0.99 & $\mathrm{C} 25-\mathrm{H} 25$ & 0.95 \\
\hline C3- & 0.99 & $\mathrm{C} 26-\mathrm{H} 26 \mathrm{~A}$ & 0.98 \\
\hline $\mathrm{C} 4-\mathrm{H} 4 \mathrm{~A}$ & 0.99 & $\mathrm{C} 26-\mathrm{H} 26 \mathrm{~B}$ & 0.98 \\
\hline $\mathrm{C} 4-\mathrm{H} 4 \mathrm{~B}$ & 0.99 & $\mathrm{C} 26-\mathrm{H} 26 \mathrm{C}$ & 0.98 \\
\hline $\mathrm{O} 1-\mathrm{P} 1-\mathrm{C} 10$ & $101.1(3)$ & $\mathrm{C} 12-\mathrm{C} 11-\mathrm{C} 10$ & $120.3(6)$ \\
\hline $\mathrm{O} 1-\mathrm{P} 1-\mathrm{S} 3$ & $118.4(2)$ & $\mathrm{C} 12-\mathrm{C} 11-\mathrm{H} 11$ & 119.9 \\
\hline $\mathrm{C} 10-\mathrm{P} 1-\mathrm{S} 3$ & $118.5(2)$ & $\mathrm{C} 10-\mathrm{C} 11-\mathrm{H} 11$ & 119.9 \\
\hline $\mathrm{O} 1-\mathrm{P} 1-\mathrm{S} 1$ & $108.6(2)$ & $\mathrm{C} 11-\mathrm{C} 12-\mathrm{C} 13$ & $119.4(6)$ \\
\hline $\mathrm{C} 10-\mathrm{P} 1-\mathrm{S} 1$ & $105.2(2)$ & $\mathrm{C} 11-\mathrm{C} 12-\mathrm{H} 12$ & 120.3 \\
\hline $\mathrm{S} 3-\mathrm{P} 1-\mathrm{S} 1$ & $104.22(11)$ & $\mathrm{C} 13-\mathrm{C} 12-\mathrm{H} 12$ & 120.3 \\
\hline $\mathrm{O} 2-\mathrm{P} 2-\mathrm{C} 20$ & $100.0(3)$ & $\mathrm{O} 3-\mathrm{C} 13-\mathrm{C} 14$ & $124.9(7)$ \\
\hline $\mathrm{O} 2-\mathrm{P} 2-\mathrm{S} 4$ & $119.4(2)$ & $\mathrm{O} 3-\mathrm{C} 13-\mathrm{C} 12$ & $114.8(6)$ \\
\hline $\mathrm{C} 20-\mathrm{P} 2-\mathrm{S} 4$ & $116.5(2)$ & $\mathrm{C} 14-\mathrm{C} 13-\mathrm{C} 12$ & $120.3(7)$ \\
\hline $\mathrm{O} 2-\mathrm{P} 2-\mathrm{S} 2$ & $108.2(2)$ & $\mathrm{C} 15-\mathrm{C} 14-\mathrm{C} 13$ & $119.2(7)$ \\
\hline $\mathrm{C} 20-\mathrm{P} 2-\mathrm{S} 2$ & $109.4(2)$ & $\mathrm{C} 15-\mathrm{C} 14-\mathrm{H} 14$ & 120.4 \\
\hline $\mathrm{S} 4-\mathrm{P} 2-\mathrm{S} 2$ & $103.04(11)$ & $\mathrm{C} 13-\mathrm{C} 14-\mathrm{H} 14$ & 120.4 \\
\hline $\mathrm{S} 2-\mathrm{S} 1-\mathrm{P} 1$ & $103.13(10)$ & $\mathrm{C} 10-\mathrm{C} 15-\mathrm{C} 14$ & $121.1(7)$ \\
\hline $\mathrm{S} 1-\mathrm{S} 2-\mathrm{P} 2$ & $105.87(10)$ & $\mathrm{C} 10-\mathrm{C} 15-\mathrm{H} 15$ & 119.5 \\
\hline $\mathrm{C} 1-\mathrm{O} 1-\mathrm{P} 1$ & $122.7(5)$ & $\mathrm{C} 14-\mathrm{C} 15-\mathrm{H} 15$ & 119.5 \\
\hline $\mathrm{C} 4-\mathrm{O} 2-\mathrm{P} 2$ & $121.7(4)$ & $\mathrm{O} 3-\mathrm{C} 16-\mathrm{H} 16 \mathrm{~A}$ & 109.5 \\
\hline $\mathrm{C} 13-\mathrm{O} 3-\mathrm{C} 16$ & $118.3(6)$ & $\mathrm{O} 3-\mathrm{C} 16-\mathrm{H} 16 \mathrm{~B}$ & 109.5 \\
\hline $\mathrm{C} 23-\mathrm{O} 4-\mathrm{C} 26$ & $115.8(6)$ & $\mathrm{H} 16 \mathrm{~A}-\mathrm{C} 16-\mathrm{H} 16 \mathrm{~B}$ & 109.5 \\
\hline $\mathrm{O} 1-\mathrm{C} 1-\mathrm{C} 2$ & $112.6(6)$ & $\mathrm{O} 3-\mathrm{C} 16-\mathrm{H} 16 \mathrm{C}$ & 109.5 \\
\hline
\end{tabular}




\begin{tabular}{|c|c|c|c|}
\hline $\mathrm{O} 1-\mathrm{C} 1-\mathrm{H} 1 \mathrm{~A}$ & 109.1 & $\mathrm{H} 16 \mathrm{~A}-\mathrm{C} 16-\mathrm{H} 16 \mathrm{C}$ & 109.5 \\
\hline $\mathrm{C} 2-\mathrm{C} 1-\mathrm{H} 1 \mathrm{~A}$ & 109.1 & $\mathrm{H} 16 \mathrm{~B}-\mathrm{C} 16-\mathrm{H} 16 \mathrm{C}$ & 109.5 \\
\hline $\mathrm{O} 1-\mathrm{C} 1-\mathrm{H} 1 \mathrm{~B}$ & 109.1 & $\mathrm{C} 25-\mathrm{C} 20-\mathrm{C} 21$ & $120.1(7)$ \\
\hline $\mathrm{C} 2-\mathrm{C} 1-\mathrm{H} 1 \mathrm{~B}$ & 109.1 & $\mathrm{C} 25-\mathrm{C} 20-\mathrm{P} 2$ & $118.2(6)$ \\
\hline $\mathrm{H} 1 \mathrm{~A}-\mathrm{C} 1-\mathrm{H} 1 \mathrm{~B}$ & 107.8 & $\mathrm{C} 21-\mathrm{C} 20-\mathrm{P} 2$ & $121.7(5)$ \\
\hline $\mathrm{C} 1-\mathrm{C} 2-\mathrm{C} 3$ & $115.8(6)$ & $\mathrm{C} 20-\mathrm{C} 21-\mathrm{C} 22$ & $119.8(6)$ \\
\hline $\mathrm{C} 1-\mathrm{C} 2-\mathrm{H} 2 \mathrm{~A}$ & 108.3 & $\mathrm{C} 20-\mathrm{C} 21-\mathrm{H} 21$ & 120.1 \\
\hline $\mathrm{C} 3-\mathrm{C} 2-\mathrm{H} 2 \mathrm{~A}$ & 108.3 & $\mathrm{C} 22-\mathrm{C} 21-\mathrm{H} 21$ & 120.1 \\
\hline $\mathrm{C} 1-\mathrm{C} 2-\mathrm{H} 2 \mathrm{~B}$ & 108.3 & $\mathrm{C} 21-\mathrm{C} 22-\mathrm{C} 23$ & $119.4(6)$ \\
\hline $\mathrm{C} 3-\mathrm{C} 2-\mathrm{H} 2 \mathrm{~B}$ & 108.3 & $\mathrm{C} 21-\mathrm{C} 22-\mathrm{H} 22$ & 120.3 \\
\hline $\mathrm{H} 2 \mathrm{~A}-\mathrm{C} 2-\mathrm{H} 2 \mathrm{~B}$ & 107.4 & $\mathrm{C} 23-\mathrm{C} 22-\mathrm{H} 22$ & 120.3 \\
\hline $\mathrm{C} 2-\mathrm{C} 3-\mathrm{C} 4$ & $115.5(6)$ & $\mathrm{O} 4-\mathrm{C} 23-\mathrm{C} 24$ & $125.0(6)$ \\
\hline $\mathrm{C} 2-\mathrm{C} 3-\mathrm{H} 3 \mathrm{~A}$ & 108.4 & $\mathrm{O} 4-\mathrm{C} 23-\mathrm{C} 22$ & $114.6(6)$ \\
\hline $\mathrm{C} 4-\mathrm{C} 3-\mathrm{H} 3 \mathrm{~A}$ & 108.4 & $\mathrm{C} 24-\mathrm{C} 23-\mathrm{C} 22$ & $120.3(6)$ \\
\hline $\mathrm{C} 2-\mathrm{C} 3-\mathrm{H} 3 \mathrm{~B}$ & 108.4 & $\mathrm{C} 23-\mathrm{C} 24-\mathrm{C} 25$ & $120.0(6)$ \\
\hline $\mathrm{C} 4-\mathrm{C} 3-\mathrm{H} 3 \mathrm{~B}$ & 108.4 & $\mathrm{C} 23-\mathrm{C} 24-\mathrm{H} 24$ & 120 \\
\hline $\mathrm{H} 3 \mathrm{~A}-\mathrm{C} 3-\mathrm{H} 3 \mathrm{~B}$ & 107.5 & $\mathrm{C} 25-\mathrm{C} 24-\mathrm{H} 24$ & 120 \\
\hline $\mathrm{O} 2-\mathrm{C} 4-\mathrm{C} 3$ & $104.8(6)$ & $\mathrm{C} 20-\mathrm{C} 25-\mathrm{C} 24$ & $120.4(7)$ \\
\hline $\mathrm{O} 2-\mathrm{C} 4-\mathrm{H} 4 \mathrm{~A}$ & 110.8 & $\mathrm{C} 20-\mathrm{C} 25-\mathrm{H} 25$ & 119.8 \\
\hline $\mathrm{C} 3-\mathrm{C} 4-\mathrm{H} 4 \mathrm{~A}$ & 110.8 & $\mathrm{C} 24-\mathrm{C} 25-\mathrm{H} 25$ & 119.8 \\
\hline $\mathrm{O} 2-\mathrm{C} 4-\mathrm{H} 4 \mathrm{~B}$ & 110.8 & $\mathrm{O} 4-\mathrm{C} 26-\mathrm{H} 26 \mathrm{~A}$ & 109.5 \\
\hline $\mathrm{C} 3-\mathrm{C} 4-\mathrm{H} 4 \mathrm{~B}$ & 110.8 & $\mathrm{O} 4-\mathrm{C} 26-\mathrm{H} 26 \mathrm{~B}$ & 109.5 \\
\hline $\mathrm{H} 4 \mathrm{~A}-\mathrm{C} 4-\mathrm{H} 4 \mathrm{~B}$ & 108.9 & $\mathrm{H} 26 \mathrm{~A}-\mathrm{C} 26-\mathrm{H} 26 \mathrm{~B}$ & 109.5 \\
\hline $\mathrm{C} 15-\mathrm{C} 10-\mathrm{C} 11$ & $119.8(7)$ & $\mathrm{O} 4-\mathrm{C} 26-\mathrm{H} 26 \mathrm{C}$ & 109.5 \\
\hline $\mathrm{C} 15-\mathrm{C} 10-\mathrm{P} 1$ & $118.9(5)$ & $\mathrm{H} 26 \mathrm{~A}-\mathrm{C} 26-\mathrm{H} 26 \mathrm{C}$ & 109.5 \\
\hline $\mathrm{C} 11-\mathrm{C} 10-\mathrm{P} 1$ & $121.2(5)$ & $\mathrm{H} 26 \mathrm{~B}-\mathrm{C} 26-\mathrm{H} 26 \mathrm{C}$ & 109.5 \\
\hline $\mathrm{C} 10-\mathrm{P} 1-\mathrm{O} 1-\mathrm{C} 1$ & $-156.0(5)$ & $\mathrm{O} 3-\mathrm{C} 13-\mathrm{C} 14-\mathrm{C} 15$ & $-179.6(6)$ \\
\hline $\mathrm{S} 3-\mathrm{P} 1-\mathrm{O} 1-\mathrm{C} 1$ & $-24.8(6)$ & $\mathrm{C} 12-\mathrm{C} 13-\mathrm{C} 14-\mathrm{C} 15$ & $-0.7(10)$ \\
\hline $\mathrm{S} 1-\mathrm{P} 1-\mathrm{O} 1-\mathrm{C} 1$ & $93.6(5)$ & $\mathrm{C} 11-\mathrm{C} 10-\mathrm{C} 15-\mathrm{C} 14$ & $-0.2(10)$ \\
\hline $\mathrm{C} 20-\mathrm{P} 2-\mathrm{O} 2-\mathrm{C} 4$ & $-173.9(5)$ & $\mathrm{P} 1-\mathrm{C} 10-\mathrm{C} 15-\mathrm{C} 14$ & $176.4(5)$ \\
\hline $\mathrm{S} 4-\mathrm{P} 2-\mathrm{O} 2-\mathrm{C} 4$ & $-45.6(6)$ & $\mathrm{C} 13-\mathrm{C} 14-\mathrm{C} 15-\mathrm{C} 10$ & $0.2(11)$ \\
\hline $\mathrm{S} 2-\mathrm{P} 2-\mathrm{O} 2-\mathrm{C} 4$ & $71.7(5)$ & $\mathrm{O} 2-\mathrm{P} 2-\mathrm{C} 20-\mathrm{C} 25$ & $163.4(6)$ \\
\hline $\mathrm{P} 1-\mathrm{O} 1-\mathrm{C} 1-\mathrm{C} 2$ & $-95.9(7)$ & $\mathrm{S} 4-\mathrm{P} 2-\mathrm{C} 20-\mathrm{C} 25$ & $33.1(7)$ \\
\hline $\mathrm{O} 1-\mathrm{C} 1-\mathrm{C} 2-\mathrm{C} 3$ & $-71.3(8)$ & $\mathrm{S} 2-\mathrm{P} 2-\mathrm{C} 20-\mathrm{C} 25$ & $-83.2(6)$ \\
\hline $\mathrm{C} 1-\mathrm{C} 2-\mathrm{C} 3-\mathrm{C} 4$ & $72.0(9)$ & $\mathrm{O} 2-\mathrm{P} 2-\mathrm{C} 20-\mathrm{C} 21$ & $-13.4(6)$ \\
\hline $\mathrm{P} 2-\mathrm{O} 2-\mathrm{C} 4-\mathrm{C} 3$ & $-168.1(5)$ & $\mathrm{S} 4-\mathrm{P} 2-\mathrm{C} 20-\mathrm{C} 21$ & $-143.7(5)$ \\
\hline $\mathrm{C} 2-\mathrm{C} 3-\mathrm{C} 4-\mathrm{O} 2$ & $65.1(8)$ & $\mathrm{S} 2-\mathrm{P} 2-\mathrm{C} 20-\mathrm{C} 21$ & $100.0(6)$ \\
\hline $\mathrm{O} 1-\mathrm{P} 1-\mathrm{C} 10-\mathrm{C} 15$ & $161.1(5)$ & $\mathrm{C} 25-\mathrm{C} 20-\mathrm{C} 21-\mathrm{C} 22$ & $-1.5(11)$ \\
\hline $\mathrm{S} 3-\mathrm{P} 1-\mathrm{C} 10-\mathrm{C} 15$ & $29.9(6)$ & $\mathrm{P} 2-\mathrm{C} 20-\mathrm{C} 21-\mathrm{C} 22$ & $175.3(6)$ \\
\hline $\mathrm{S} 1-\mathrm{P} 1-\mathrm{C} 10-\mathrm{C} 15$ & $-86.0(6)$ & $\mathrm{C} 20-\mathrm{C} 21-\mathrm{C} 22-\mathrm{C} 23$ & $1.1(11)$ \\
\hline $\mathrm{O} 1-\mathrm{P} 1-\mathrm{C} 10-\mathrm{C} 11$ & $-22.4(6)$ & $\mathrm{C} 26-\mathrm{O} 4-\mathrm{C} 23-\mathrm{C} 24$ & $-2.2(11)$ \\
\hline $\mathrm{S} 3-\mathrm{P} 1-\mathrm{C} 10-\mathrm{C} 11$ & $-153.5(5)$ & $\mathrm{C} 26-\mathrm{O} 4-\mathrm{C} 23-\mathrm{C} 22$ & $177.7(8)$ \\
\hline $\mathrm{S} 1-\mathrm{P} 1-\mathrm{C} 10-\mathrm{C} 11$ & $90.6(5)$ & $\mathrm{C} 21-\mathrm{C} 22-\mathrm{C} 23-\mathrm{O} 4$ & $-179.7(6)$ \\
\hline $\mathrm{C} 15-\mathrm{C} 10-\mathrm{C} 11-\mathrm{C} 12$ & $0.8(10)$ & $\mathrm{C} 21-\mathrm{C} 22-\mathrm{C} 23-\mathrm{C} 24$ & $0.2(11)$ \\
\hline $\mathrm{P} 1-\mathrm{C} 10-\mathrm{C} 11-\mathrm{C} 12$ & $-175.8(5)$ & $\mathrm{O} 4-\mathrm{C} 23-\mathrm{C} 24-\mathrm{C} 25$ & $178.9(7)$ \\
\hline $\mathrm{C} 10-\mathrm{C} 11-\mathrm{C} 12-\mathrm{C} 13$ & $-1.3(10)$ & $\mathrm{C} 22-\mathrm{C} 23-\mathrm{C} 24-\mathrm{C} 25$ & $-1.0(11)$ \\
\hline $\mathrm{C} 16-\mathrm{O} 3-\mathrm{C} 13-\mathrm{C} 14$ & $-7.2(9)$ & $\mathrm{C} 21-\mathrm{C} 20-\mathrm{C} 25-\mathrm{C} 24$ & $0.6(12)$ \\
\hline
\end{tabular}



$\mathrm{C} 16-\mathrm{O} 3-\mathrm{C} 13-\mathrm{C} 12$
$173.8(6)$
$\mathrm{P} 2-\mathrm{C} 20-\mathrm{C} 25-\mathrm{C} 24$
$-176.2(6)$
$\mathrm{C} 11-\mathrm{C} 12-\mathrm{C} 13-\mathrm{O} 3$
$-179.7(6)$
$\mathrm{C} 23-\mathrm{C} 24-\mathrm{C} 25-\mathrm{C} 20$
$0.6(12)$
$\mathrm{C} 11-\mathrm{C} 12-\mathrm{C} 13-\mathrm{C} 14$
$1.3(10)$

Hydrogen-bond geometry $\left(A,{ }^{\circ}\right)$

$\mathrm{Cg}$ is the centroid of the $\mathrm{C} 20-\mathrm{C} 25$ ring.

\begin{tabular}{lllll}
\hline$D-\mathrm{H} \cdots A$ & $D-\mathrm{H}$ & $\mathrm{H} \cdots A$ & $D \cdots A$ & $D-\mathrm{H} \cdots A$ \\
\hline $\mathrm{C} 1-\mathrm{H} 1 B \cdots \mathrm{S} 3$ & 0.99 & 2.81 & $3.3883(2)$ & 118 \\
$\mathrm{C} 4-\mathrm{H} 4 B \cdots \mathrm{O} 1$ & 0.99 & 2.48 & $3.1308(2)$ & 123 \\
$\mathrm{C} 4-\mathrm{H} 4 B \cdots \mathrm{O} 4^{\mathrm{i}}$ & 0.99 & 2.56 & $3.2708(2)$ & 128 \\
$\mathrm{C} 11-\mathrm{H} 11 \cdots \mathrm{O} 4^{\mathrm{i}}$ & 0.95 & 2.62 & $3.4951(3)$ & 154 \\
$\mathrm{C} 24-\mathrm{H} 24 \cdots \mathrm{O} 3^{\mathrm{ii}}$ & 0.95 & 2.51 & $3.4240(3)$ & 162 \\
$\mathrm{C} 16-\mathrm{H} 16 A \cdots C g^{\mathrm{iii}}$ & 0.98 & 2.62 & $3.454(8)$ & 143
\end{tabular}

Symmetry codes: (i) $x,-y+1 / 2, z+1 / 2$; (ii) $-x+1, y-1 / 2,-z+1 / 2$; (iii) $x-1,-y+1 / 2, z+1 / 2$. 